\title{
AN EFFICIENT ROUTING PROTOCOL FOR DELAY TOLERANT NETWORKS (DTNs)
}

\author{
Nandha kumar.R ${ }^{1}$,Dr.P.Varalakshmi ${ }^{2}$ and V.S.Harikannan ${ }^{3}$ \\ ${ }^{1}$ Department of Computer Technology, Anna University, Chennai, India \\ Nandhakumarr03@gmail.com \\ ${ }^{2}$ Department of Information Technology, Anna university, Chennai, India \\ varanip@gmail.com \\ ${ }^{3}$ Department of Computer Technology, Anna University, Chennai, India \\ harikannan.mit15@gmail.com
}

\begin{abstract}
Delay-Tolerant Networks are those which lacks continuous communications among mobile nodes . Distributed clustering scheme and cluster-based routing protocol are used for DelayTolerant Mobile Networks (DTMNs). The basic idea is to distributive group mobile nodes with similar mobility pattern into a cluster, which can then interchangeably share their resources for overhead reduction and load balancing, aiming to achieve efficient and scalable routing in DTMN. Load balancing is carried out in two ways, Intra cluster load balancing and Inter cluster load balancing. The Convergence and stability become major challenges in distributed clustering in DTMN. An efficient routing protocol will be provided for the delay tolerant networks through which the stability of the network is maintained.Based on nodal contact probabilities, a set of functions including Sync(), Leave(), and Join() are devised for cluster formation and gateway selection. Finally, the gateway nodes exchange network information and perform routing.
\end{abstract}

\section{KEYWORDS}

Clustering, delay-tolerant networks, replication, load balancing.

\section{INTRODUCTION}

A mobile ad-hoc network (MANET) is a self-configuring infra structure less network of mobile devices connected by wireless links. Each device in a MANET is free to move independently in any direction, and will therefore change its links to other devices frequently. Each must forward traffic unrelated to its own use, and therefore be a router. The primary challenge in building a MANET is equipping each device to continuously maintain the information required to properly route traffic. Such networks may operate by themselves or may be connected to the larger Internet.

\subsection{DELAY TOLERANT NETWORKS}

Delay Tolerant Network (DTN) is fundamentally an opportunistic communication system, where communication links only exists temporarily, rendering it impossible to establish end-to-end connections for data delivery. End to end communication between source to destination can be achieve by disjoint the nodes into different group. Connectivity delay, asymmetric data and high data rates are overcome by delay tolerant networks. DTN operate above all other existing protocol

Natarajan Meghanathan, et al. (Eds): SIPM, FCST, ITCA, WSE, ACSIT, CS \& IT 06, pp. 573-578, 2012. (C) CS \& IT-CSCP 2012

DOI : $10.5121 /$ csit.2012.2356 
stack and provide gateway function between nodes when data transmitted between different networks or different group inside the networks. When groups are formed depend on their contact probability of the nodes. Each of these networking environments has their own specialized protocol stack and gateway are responsible for storing message, when reliable delivery is required.

The basic idea for distributed clustering scheme to reduce overhead due to buffer space through load balancing. Grouping mobile nodes with similar mobility pattern increases nodal contact probability among mobile nodes within the group, which can interchange their resource. Due to the lack of continuous communications and possible errors in the estimation of the nodal contact probability, convergence and stability become major challenges in such cluster formation. Finally the gateway node exchange network information and performing routing[10].

\subsection{CLUSTER FORMATION}

Cluster head selection in DTNs is done based on the nodal probabilities of the nodes. The node with highest nodal contact probability (threshold probability) is taken as the cluster head. Data from one cluster to another will be transmitted through the gateway nodes. Gateway nodes in DTNs are selected based on the nodal contact probability of nodes I one cluster with the nodes in the other cluster. A node in the same cluster transmits data from one node to another with their transmission range of all the nodes within the cluster. Nodes in the different cluster transmits data to another cluster depend upon the probability of nodal connectivity; direct transmission is also employed data from one node to another. Every gateway node constructs a cluster connectivity packet and distributes it to other gateway in the networks. The cluster connectivity of the gateway comprises its cluster ID and a list of cluster to which it serve as gateway along with corresponding contact probability. Once the routing table is obtained, the routing is performed from a cluster to another cluster through single hop Inter cluster routing and Intra cluster routing.

\subsection{LOAD BALANCING}

Load balancing is an effective enhancement to the proposed routing protocol. The basic idea is to share traffic load among cluster members in order to reduce the dropping probability due to queue overflow at some nodes. Sharing traffic inside a cluster is reasonable, because nodes in the same cluster have similar mobility pattern, and thus similar ability to deliver data messages. Whenever the queue length of a node exceeds a threshold, denoted by $\Lambda$, it starts to perform load balancing. More specifically, it randomly transmits as many messages as possible to any node it meets, until their queues are equally long or the latter's queue becomes longer than $\Lambda$. Load Balancing in a cluster and among the clusters are done in either ways of Intra cluster load balancing ,Inter cluster load balancing.

\section{LITERATURE SURVEY}

Clusters are formed based on their nodal contact probabilities the probability of nodes meeting each other. Based on their nodal contact probability the threshold probability will be calculated, using which the clusters are formed and the gateways nodes are selected to route data from one cluster to another[1][2].

In [9] capacity and delay trade off mechanism, the capacity of the cell partitioned networks and analysis the delay of the capacity achieving relay algorithm. The packet are transmitted and routed according to the timeslot assign to each node without violating the physical constrains of the partitioned cell. The capacity region depends only on the steady-state user location distributaries. Hence, any markovian model of the user mobility which in steady state distribute users independently and the network yields uniformly over the same expression for mobile nodes. 
A cluster-based self-organizing strategy is proposed for building a backbone among the mobile devices, detecting segmentation, and recovery [3][4]. In this approach, each mobile device is controlled by a multi-role agent, which performs these tasks efficiently based only on local interactions; role management allows the backbone reconfiguration when the nodes leave or arrive to the network yielding a complex global emergent behaviour.[5] Energy saving is achieved by adapting the time interval and power of transmission after the network formation. The inconsistency problem exist both in member and gateway nodes. When two nodes in the same cluster may have two different gateways to another cluster in figure 1. A node may lose its gateway to an adjacent cluster because the gateway node has left. These inconsistency problem employing by synchronization mechanism where nodes exchange and keep only the most up to data information. The replication mechanism that routing protocols adopt to ensure delivery of the original packet to the sink is to transmit multiple copies of the same packet over different paths in order to recover from some path failures

Wireless networks are without a doubt one of the central issues in current research topics due to the harsh environmental conditions in which such networks can be deployed and their unique network characteristics, specifically limited power supply, processing and communication capabilities[6]. [7] Presented with many challenges and design issues that affect the data routing, a need for a fault tolerant routing protocol becomes essential. An algorithm to form the various paths from sender to destination will be provide [8][9].

\section{PROPOSED WORK}

Scalability is one of the main problem in DTN networks, over population of cluster lead to the instability .So, fault tolerance techniques are used to solve scalability problem .Data in nodes are transmitted through gateway nodes and errors in gateway nodes are solved using fault tolerance techniques . Data loss during transmission due to weak signal strength and intermittent transmission capabilities in figure 2. In delay tolerant network data lost will be much higher when bandwidth limits among the node is much low. so we have chosen some techniques to apply in this routing protocol to prevent the data loss in routing .Load balancing is an effective enhancement to the proposed routing protocol. The basic idea is to share traffic load among cluster members in order to reduce the dropping probability due to queue overflow at some nodes. Specifically, a nodes performs load balancing when its queue exceed their threshold limit, by randomly transmitting as many packets as possible to any node which meet their queue limit and with their contact probability region. Sharing traffic inside a cluster is reasonable, because nodes in the same cluster have similar mobility pattern, and thus similar ability to deliver data messages.

An efficient routing protocol will be provided for the delay tolerant networks through which the stability of the network is maintained.

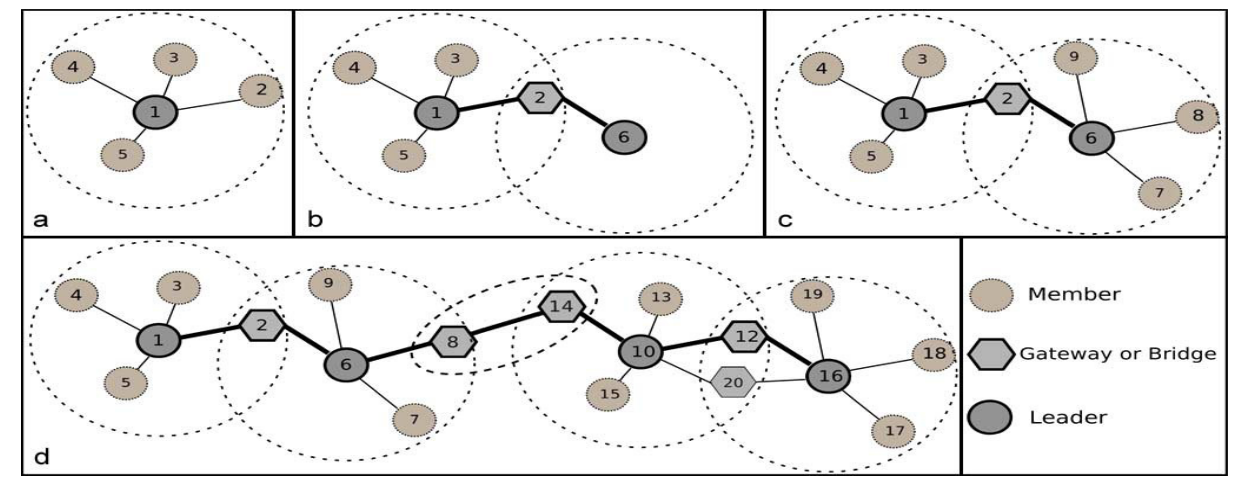

Figure 1: Topology diagram 


\title{
ALGORITHM:
}

i. Head and Gateway selection

If(Neigh!0)

$$
\begin{aligned}
& \text { (leaders }==0) \text { then } \\
& \text { ROLE=leader }
\end{aligned}
$$

Else (there exist atleast one leader )

If(ROLE=leader) then

Solveconflict();

Else (my role is not leader )

If(leaders $==1$ )

ROLE $=$ member

Verify_consistency();

Else (there exists more than one leader)

ENDIF

ROLE=gateway;

ENDIF

ELSE

ENDIF

ENDIF

ROLE $=$ any node _member

ii. Intra Cluster Load Balancing

MAKE_WEIGHT_LIST()

SORT(WEIGHT_LIST)

FOR ( All nodes Contacting with Cluster head )

IF ( CONTACT PROBABILITY OF CLUSTER $<$ THRESHHOLD

PROBABILITY OF CLUSTER )

THEN

FOR ( all nodes in Weighted List )

DELETE those nodes from WEIGHT_LIST

FIND_AVERAGE_WEIGHTED_PROBABILY

\author{
IF( AVERAGE_WEIGHTED_PROBABILITY > CONTACT \\ PROBABILITY OF NODE ) \\ THEN
}

\section{REPLICATE()}

DELETE those nodes from WEIGHT_LIST

Transfer all the Data messages to Node Available in WEIGHT_LIST

iii. Inter Cluster Load Balancing

IF( TRAFFIC is not reduced after applying INTRA_LOAD_BALANCING) THEN

CHOOSE_ADJUCENT_CLUSTER()

START_INTER_CLUSTER_LOAD_BALANCING()

IF ( AVERAGE_WEIGHTED PROBABILITY OF Present cluster < AVERAGE_WEIGHTED PROBABILITY NODE in Adjacent Cluster $\& \&$ NUMBER OF NODE IN Adjacent Cluster > NUMBER OF NODE

THEN in Present Cluster )

Transfers data to those cluster and

Apply INTRA_CLUSTER_LOAD_BALANCING() in Adjacent Cluster 


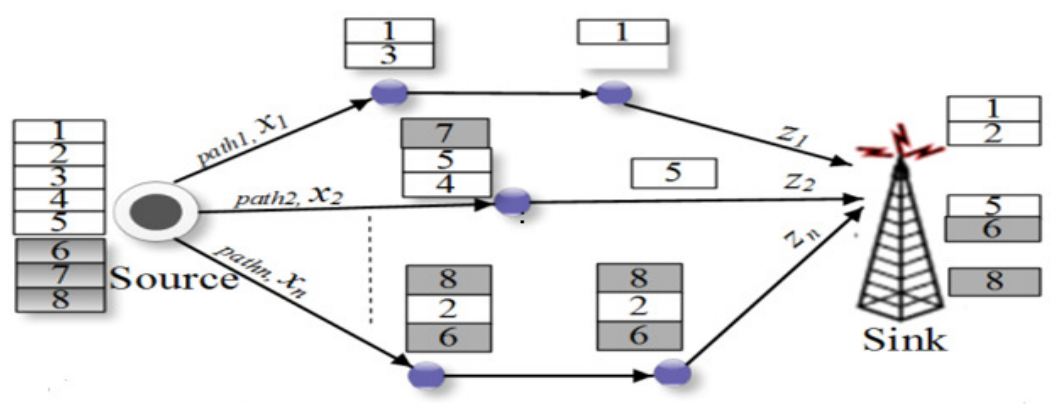

\section{Data fragments \\ Parity fragments}

Figure 2: Data Transmission

\section{Performance analysis}

In the existing project data dropping probability average values will be always more than 50 percentages. But when we are applying the algorithms that have been discussed above data dropping probability value will be reduced to below 50 percentages in figure 3 . so that we can minimize the data dropping value with using this cluster based routing environment. Every node has the maximum queue size, which varies from 20 to 100 during the simulation. Our nodal contact based on similar mobility pattern among mobile nodes increases overall delivery ratio. The delivery ratio due to load balancing, when the data or message in the queue almost full. It transmit data to other members inside the group, resulting in low drop rate at each single node.

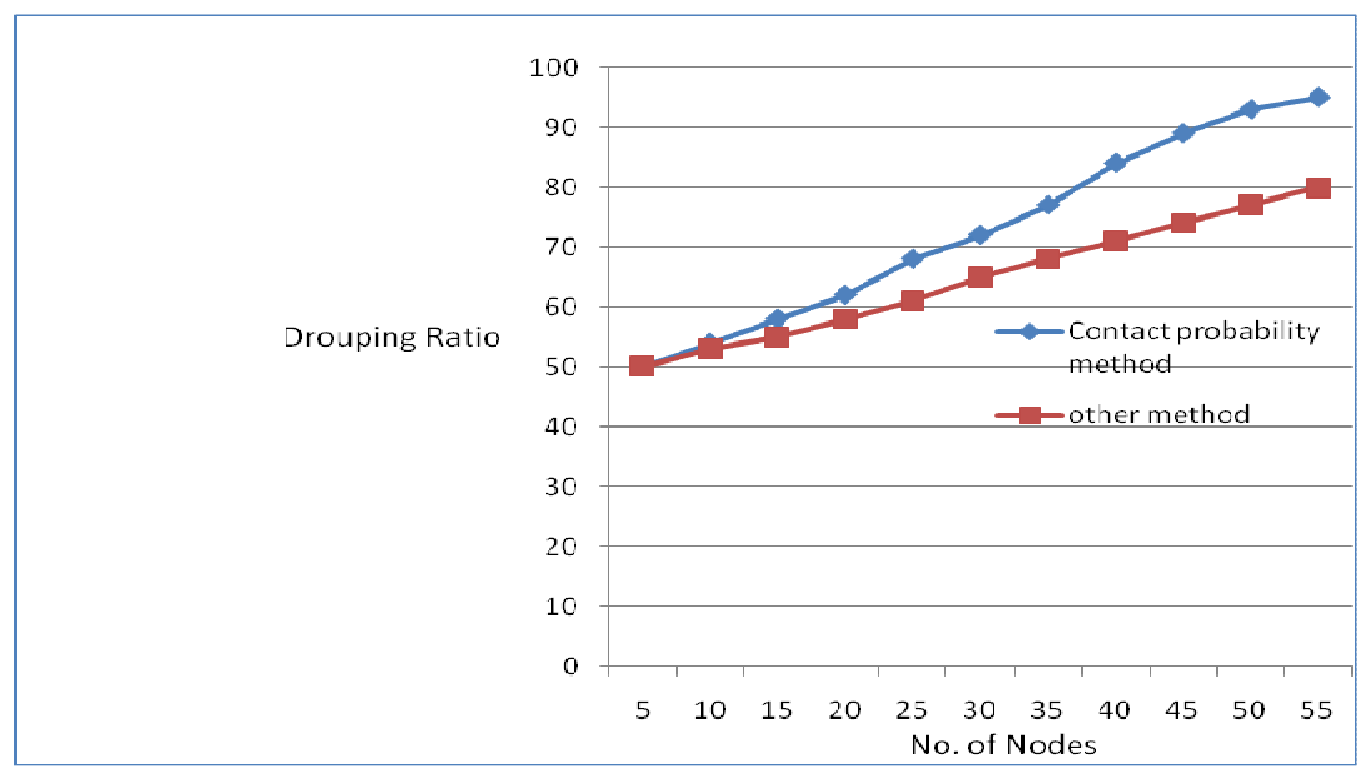

Figure 3 : Data dropping values at various stages 


\section{CONCLUSION}

An efficient routing protocol has been provided for the delay tolerant networks through which the stability of the network is maintained. Another important consideration taken into account is load balancing which is implemented using grouping of mobile nodes with similar mobility pattern techniques either retransmission or replication data to their neighbour nodes. Nodal contact probability ratio or threshold is maintained in each group head to achieve better stability and increase scalability among mobile nodes.

\section{REFERENCES}

[1] Ha Dang and Hongyi Wu "Clustering and Cluster-Based Routing Protocol for Delay-Tolerant Mobile Networks,” IEEE Transactions on Wireless Communication , vol. 9, no. 6, pp. 174-185 ,June 2010.

[2] Devices J. Guadalupe Olascuaga-Cabrera, Ernesto López-Mellado, Andres Mendez-Vazquez, and Félix Francisco Ramos-Corchado, "A Self-Organization Algorithm for Robust Networking of Wireless", IEEE Journals, vol 11,no 3 pp 102-117,March 2011.

[3] Ning Li and Jennifer C. Hou "Localized Fault-Tolerant Topology Control in Wireless Ad Hoc Networks," IEEE Transactions on Distributed Systems ,vol 17,no 4 pp 601-611 April ,2010.

[4] Hind Alwan and Anjali Agarwal "A Survey on Fault Tolerant Routing Techniques in Wireless networks," Third International Conference on Sensor Technologies and Applications, vol 12,no 5,pp 201-217,March ,2009.

[5] Che-Aron, Z, Al-Khateeb, W. F. M and Anwar.F "An Enhancement of Fault-Tolerant Routing Protocol for Wireless Network," Malaysia, Kuala Lumpur, Malaysia, International Conference on Computer and Communication Engineering, vol 15,no.7,pp 607-612 ,May 2010.

[6] Y. Wang and H. Wu, "DFT-MSN The Fault tolerant mobile sensor network for pervasive information gathering," in Proc. 26th Annual Joint Conference of the IEEE Computer and Communications Societies, IEEE,vol 4,no.7 pp 401-409,February 2010.

[7] K. Fall, "Dynamic Cluster Header Selection and Conditional Re-clustering for Wireless Sensor Networks" in Proc.ACM SIGCOMM, vol .9,no.4,pp. 27-34, 2010.

[8] S. Burleigh, A. Hooke, L. Torgerson, K. Fall, V. Cerf, B. Durst,K. Scott, and H. Weiss, "Clustering and fault tolerance for target tracking using wireless sensor networks," IEEE Communication. Mag., vol. 41, no. 6, pp. 128-136, 2010.

[9] Michael J.Neely and Eytan Modiano, "Capacity and Delay Tradeoffs for Ad Hoc Mobile Networks," in IEEE Transactions on Information Theory, Vol. 51, No. 6,pp. 1917-1937, June 2005.

[10] Ha Dang and Hongi Wu,"Clustering Based Data Transmission protocol in Delay Tolerant Mobile Networks," IEEE conference, vol. 01, pp.529-530, 2008.

[11] T. Small and Z. J. Haas, "An Intrusion Detection Architecture for Clustered Wireless Ad Hoc Networks," in Proc. MobiHOC, IEEE,vol.12,no.9,pp. 233- 244, 2010.

[12] M.Grossglauser and D.N.C Tse,"Mobility increases the capacity of ad-hoc wireless networks," in Proc. IEEE INFOCOM, Anchorage, AK, PP. 1360-1369., April 2001.

[13] Y.Wang, H. Wu, F. Lin and N.F.Tzeng,"Protocol Design and Optimization for Delay/Fault-Tolerant Mobile Sensor Networks, " in Proc. Of ICDCS, PP.7, 2007. 